\title{
Transplantation after Mustard operation for transposition of the great arteries
}

\author{
Shintaro Katahira ${ }^{1}$, Yukiharu Sugimura ${ }^{1}$, Hug Aubin $^{1}$, Hayato Ise ${ }^{1}$, Yoshikatsu Saiki ${ }^{2}$, \\ Artur Lichtenberg ${ }^{3}$, Ralf Westenfeld ${ }^{1}$, Udo Boeken ${ }^{1}$, and Payam Akhyari ${ }^{1}$ \\ ${ }^{1}$ Heinrich-Heine-Universitat Dusseldorf \\ ${ }^{2}$ Tohoku University \\ ${ }^{3}$ University Hospital Dusseldorf
}

December 15, 2020

\begin{abstract}
As long-term outcomes of congenital heart diseases improve, the probability of adult patients presenting for heart transplantation for late failure of congenitally corrected heart disease also increases. In patients with dextro-transposition of the great arteries (d-TGA) who were initially treated in the era of Mustard or Senning procedures and before Jatene procedure was introduced, progressive systemic right ventricular failure represents a problem in the very long-term follow-up. We report a rare case of heart transplantation as a third operation 36 years after Mustard procedure in a patient with d-TGA experiencing late failure of the systemic right ventricle.
\end{abstract}

\section{Introduction}

In dextro-transposition of the great arteries (d-TGA), the Jatene procedure was introduced in 1975, resulting in greatly improved outcomes ${ }^{1}$. However, systemic right ventricular (sRV) failure in adulthood becomes a problem in earlier types of correction, e.g., Mustard or Senning procedure ${ }^{2}$. In the latter patient cohort, heart transplantation (HTX) is the only treatment for sRV failure. Intraoperative identification and orientation regarding the atrial chambers, as well as anatomic variation with respect to the position of the great arteries, represent technical challenges during HTX. Current literature contains few narrative reports on HTX after Mustard procedure ${ }^{3,4}$. Here, we demonstrate a case along with a surgical video of successful HTX after Mustard operation.

\section{Case Report}

A 36-year-old man with d-TGA and Mustard procedure at 15 months after birth and second operation with augmentation plasty for SVC stenosis at the age of 6 years was admitted to the emergency department for heart failure (HF) symptoms. On echocardiography, sRV was dilated (end-diastolic dimension 56mm) with a severely impaired ejection fraction (EF) of $15 \%$ and moderate regurgitation at systemic atrioventricular valve. Preoperative computed tomography showed anterior location of the aorta, regular anatomic relation of the Mustard baffle in the systemic atrium in close anatomic relation to the pulmonary valve, moreover the anterior wall of the systemic ventricle grossly adhering to the dorsal aspect of the sternum (Fig. $1 \mathrm{~A}, \mathrm{~B}$ ). Cardiac index was $1.65 \mathrm{l} / \mathrm{min}^{*} \mathrm{~m}^{2}$ and initial lactate was $1.6 \mathrm{mmol} / \mathrm{l}$. The patient was admitted to intensive care and high urgency (HU) status was granted. Fifty-two days after HU status, HTX was performed 35 years after Mustard procedure.

The operative strategy included femoral cannulation and cardiopulmonary bypass (CPB) initiation prior to re-sternotomy due to expected adhesions to sRV. After partial dissection of the adhesions, the systemic 
atrium was vented to avoid pulmonary congestion during further preparation and manipulation. Aortic clamping was performed early to avoid air embolism, and both caval veins were incised for later bicaval implantation of donor heart. Next, systemic (i.e., anatomic right) atrium was opened and Mustard baffle as well as pulmonary vein (PV) ostia were identified from the endocardial side as well as PV location from outside of the atrium (Fig.2). Aorta and pulmonary trunk were transected more distally than in regular HTX to achieve a more regular anatomic relation. The resulting recipient dimension of the aorta was remarkably small. Due to the specific technique of Mustard correction, the inter-PV distance revealed to be relatively small and the anatomic left atrial cuff limited in size when compared to the common anatomy in HF patients. Therefore, when excising recipient heart, the incision line was performed as much as possible distant from the $\mathrm{PV}$ ostia. Addition perpendicular incision of the remaining left atrial cuff was performed in between the two left PV ostia as well as caudally and cranially in between the left and right atrial PV ostia in order to enlarge the anastomotic line on the recipient side. As a further modification on the donor side, cardiac graft was harvested with a long segment of the aorta, including most part of the aortic arch. HTX was performed by bicaval method and the anastomosis was performed in the order of left atrium, IVC, SVC, pulmonary artery, ascending aorta. Despite the more liberal excision of recipient great arteries, the distal ascending aorta was yet located anteriorly and slightly left to the normal anatomy. Utilizing longer segments of the donor graft and more distal anastomotic lines, it was possible to perform both anastomoses of great arteries without the use of prosthetic materials. The aortic anastomosis was further complicated by a remarkable size mismatch but proved to be feasible without prosthetic material. Total donor heart ischemia time was 214 minutes. After 131 min of reperfusion, weaning from CPB was performed with moderate doses of catecholamines, inhalative nitric oxide, and intermittent inhalative prostacyclin therapy. The patient was extubated on $1^{\text {st }}$ postoperative day and further postoperative course was unremarkable. There was no particular problem with postoperative echocardiography, and he was discharged on the $33^{\text {rd }}$ postoperative day without any other complications.

\section{Discussion}

In the case of d-TGA, Mustard procedure was performed before Jatene procedure ${ }^{2}$, providing with progressive failure of the sRV resulting late after surgery. Implantable ventricular assist device (VAD) therapy has been reported in the latter scenario, however, with mixed results of VAD in sRV, which may suggest to favor HTX as the first choice in this particular patient cohort ${ }^{5}$.

Beyond pre-operative stabilization and postoperative management, intraoperative technical issues represent important components for successful treatment of d-TGA patients with failing systemic ventricle. Anatomical abnormalities represent a considerable challenge, particularly after previous operations. A thorough diagnostic workup utilizing modern imaging modalities for precise localization of native structures (e.g., course and ostia of PVs) and reconstructive implant material (e.g., atrial baffle) should be obtained to improve the quality of preoperative decision finding regarding operative strategy. Although not experienced in this case, further reconstruction steps may be necessary for complex anatomic scenarios to enable HTX, e.g., using a baffle or vascular grafts ${ }^{3}$.

The point devised in the surgery of this case was the creation of the left atrial anastomosis. Since the adhesion between the right atrium and PV was severe, ascending aorta was clamped, the right atrium was incised to confirm the left atrium and PV from the atrium and pericardium, and the heart can be removed safely without injury. In this case, IVC and SVC were located on the right side, no special reconstruction was required, and reconstruction with the normal bicaval method was possible.

\section{Ethical approval:}

This manuscript followed the principles of the Declaration of Helsinki and the Declaration of Istanbul. The appropriate informed consent was obtained for the publication of this manuscript.

\section{Data availability statement:}

Data sharing is not applicable to this article as no new data were created or analyzed in this study. 


\section{References}

1. Jatene AD, Fontes VF, Paulista PP, et al. Anatomic correction of transposition of the great vessels. $J$ Thorac Cardiovasc Surg.1976;72(3):364-370.

2. Kiener A, Kelleman M, McCracken C, Kochilas L, St Louis JD, Oster ME. Long-Term Survival After Arterial Versus Atrial Switch in d-Transposition of the Great Arteries. Ann Thorac Surg.2018;106(6):18271833.

3. Munoz-Guijosa C, Ginel A, Montiel J, Padro JM. Orthotopic heart transplantation in a patient with situs invs, transposition of the great arteries and Mustard operation. Eur J Cardiothorac Surg.2008;34(1):219-221.

4. Messner GN, Gregoric ID, Chu T, et al. Orthotopic heart transplantation in a patient with D-transposition of the great arteries: after a Mustard procedure. Tex Heart Inst J. 2005;32(4):541-543.

5. Maly J, Netuka I, Besik J, Dorazilova Z, Pirk J, Szarszoi O. Bridge to transplantation with long-term mechanical assist device in adults after the Mustard procedure. J Heart Lung Transplant.2015;34(9):11771181.

\section{Figure Legends}

Fig. 1. Preoperative enhanced computed tomography

(A) Frontal view demonstrates that blood flow of SVC and IVC are re-directed through the baffle towards the mitral valve and the LV connected with PA. (B) Sagittal view shows that the systemic RV and aorta are located anteriorly with respect to the pulmonary ventricle and the pulmonary trunc. PA, pulmonary artery; LV, left ventricle; IVC, inferior vena cava; SVC, superior vena cava; RV; right ventricle.

Fig. 2. Intraoperative imaging

When the anatomic right atrium, i.e., systemic atrium, was incised, the Mustard baffle became immediately identified and LA and PV could be confirmed underneath in the depth of the systemic atrial chamber. LA, left atrium; LUPV, left upper pulmonary vein; LLPV, left lower pulmonary vein; RUPV, right upper pulmonary vein; RLPV, right lower pulmonary vein

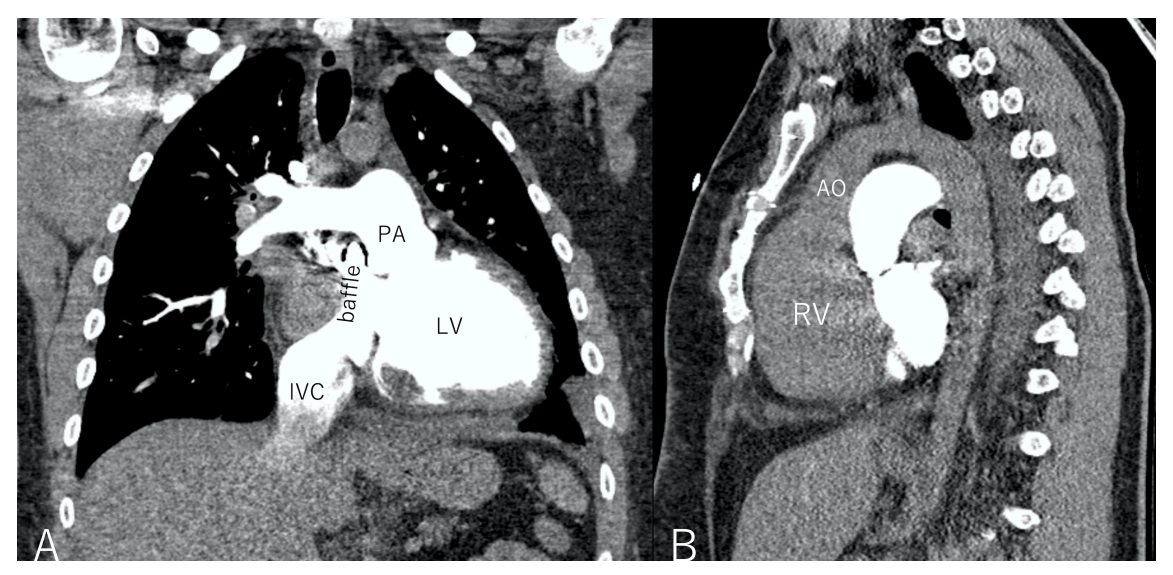




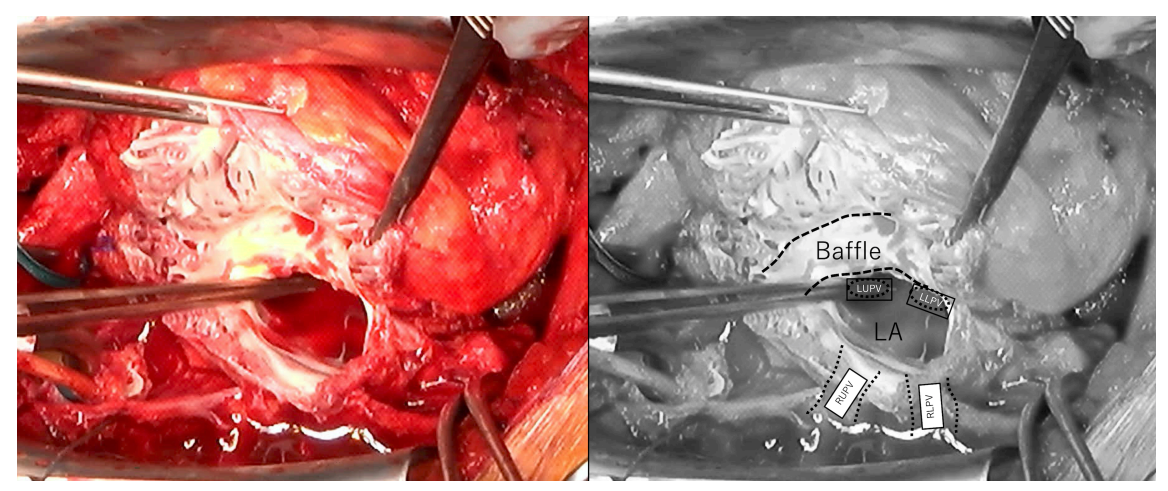

